\title{
Gene expression and in situ protein profiling of candidate SARS-CoV-2 receptors in human airway epithelial cells and lung tissue
}

\author{
Jennifer A. Aguiar ${ }^{1}$, Benjamin J-M. Tremblay ${ }^{1}$, Michael J. Mansfield ${ }^{2}$, \\ Owen Woody ${ }^{3}$, Briallen Lobb (1) 1 , Arinjay Banerjee ${ }^{4}$, Abiram Chandiramohan $^{5}$, \\ Nicholas Tiessen ${ }^{5}$, Quynh Cao ${ }^{5}$, Anna Dvorkin-Gheva $\mathbb{1}^{4}$, Spencer Revill ${ }^{5}$, \\ Matthew S. Miller ${ }^{4,6,7}$, Christopher Carlsten ${ }^{8}$, Louise Organ ${ }^{9}$, Chitra Joseph ${ }^{9}$, \\ Alison John ${ }^{9}$, Paul Hanson ${ }^{10}$, Richard C. Austin ${ }^{11}$, Bruce M. McManus ${ }^{10}$, \\ Gisli Jenkins', Karen Mossman $\mathbb{1}^{4}$, Kjetil Ask $\mathbb{1}^{4,5}$, Andrew C. Doxey ${ }^{1,5,12}$ and \\ Jeremy A. Hirota ${ }^{1,4,5,6,12}$
}

@ERSpublications

ACE2 gene and protein expression is low to absent in airway and alveolar epithelial cells in human lungs. This study suggests the presence of a mechanism dynamically regulating ACE2 expression in human lung or other receptors for SARS-CoV-2. https://bit.ly/3f85R1I

Cite this article as: Aguiar JA, Tremblay BJ-M, Mansfield MJ, et al. Gene expression and in situ protein profiling of candidate SARS-CoV-2 receptors in human airway epithelial cells and lung tissue. Eur Respir J 2020; 56: 2001123 [https://doi.org/10.1183/13993003.01123-2020].

ABSTRACT In December 2019, severe acute respiratory syndrome coronavirus 2 (SARS-CoV-2) emerged, causing the coronavirus disease 2019 (COVID-19) pandemic. SARS-CoV, the agent responsible for the 2003 SARS outbreak, utilises angiotensin-converting enzyme 2 (ACE2) and transmembrane serine protease 2 (TMPRSS2) host molecules for viral entry. ACE2 and TMPRSS2 have recently been implicated in SARS-CoV-2 viral infection. Additional host molecules including ADAM17, cathepsin L, CD147 and GRP78 may also function as receptors for SARS-CoV-2.

To determine the expression and in situ localisation of candidate SARS-CoV-2 receptors in the respiratory mucosa, we analysed gene expression datasets from airway epithelial cells of 515 healthy subjects, gene promoter activity analysis using the FANTOM5 dataset containing 120 distinct sample types, single cell RNA sequencing (scRNAseq) of 10 healthy subjects, proteomic datasets, immunoblots on multiple airway epithelial cell types, and immunohistochemistry on 98 human lung samples.

We demonstrate absent to low ACE2 promoter activity in a variety of lung epithelial cell samples and low ACE2 gene expression in both microarray and scRNAseq datasets of epithelial cell populations. Consistent with gene expression, rare ACE2 protein expression was observed in the airway epithelium and alveoli of human lung, confirmed with proteomics. We present confirmatory evidence for the presence of TMPRSS2, CD147 and GRP78 protein in vitro in airway epithelial cells and confirm broad in situ protein expression of CD147 and GRP78 in the respiratory mucosa.

Collectively, our data suggest the presence of a mechanism dynamically regulating ACE2 expression in human lung, perhaps in periods of SARS-CoV-2 infection, and also suggest that alternative receptors for SARS-CoV-2 exist to facilitate initial host cell infection.

This article has supplementary material available from erj.ersjournals.com

Received: 10 April 2020 | Accepted after revision: 1 July 2020

Copyright OERS 2020. This version is distributed under the terms of the Creative Commons Attribution NonCommercial Licence 4.0. 


\section{Introduction}

In 2003, the severe acute respiratory syndrome (SARS) outbreak caused by the SARS coronavirus (SARS-CoV) resulted in 8096 probable cases with 774 confirmed deaths [1, 2]. In patients with SARS, deaths were attributed to acute respiratory distress associated with diffuse bilateral pneumonia and alveolar damage [3]. In December 2019, SARS-CoV-2 emerged, causing the coronavirus disease 2019 (COVID-19) pandemic. SARS-CoV-2 is spreading at a much more rapid rate than SARS-CoV [4-6]. Similar clinical reports of diffuse bilateral pneumonia and alveolar damage have been reported [7-9]. Severe cases of SARS-CoV-2 have been associated with infections of the lower respiratory tract, with detection of the virus throughout this tissue as well as the upper respiratory tract [7-9]. The biological mechanisms that may govern differences in the number of SARS and COVID-19 cases remain undefined. It is possible that SARS-CoV-2 possesses distinct molecular mechanisms that affect the virulence through viral proteins, greater susceptibility of host cells to infection, permissivity of host cells to virus replication, or some combination of these and other potentially unknown factors [10-13]. Understanding SARS-CoV and SARS-CoV-2 virus similarities and differences at the molecular level in the host may provide insights into transmission, pathogenesis and interventions.

The seminal report identifying the receptor for SARS-CoV used a HEK293 cell over-expression system to identify angiotensin-converting enzyme 2 (ACE2) as a receptor by co-immunoprecipitation with SARS-CoV spike domain 1 [14]. Subsequently, spike protein of SARS-CoV was identified as the viral interacting partner of ACE2. Host protease activity by transmembrane serine protease 2 (TMPRSS2) facilitates ACE2 ectodomain cleavage and fusion of SARS-CoV membrane with host cell membrane [1517]. ADAM17 (ADAM metallopeptidase domain 17, a member of the ADAM (a disintegrin and metalloprotease domain) family) has also been demonstrated to cleave ACE2 ectodomain, but this was not required for SARS-CoV infection [18-20]. Mechanisms of SARS-CoV entry distinct from ACE2 have also been reported and include activation by endosomal cathepsin L and cell surface expression of CD147 (also known as basigin (BSG)) or GRP78 (78-kDa glucose-regulated protein; also known as heat shock protein family A (Hsp70) member 5 (HSPA5)) [21-23]. Each of these receptors was mechanistically interrogated and results suggested that SARS-CoV could initiate host cell entry and infection using multiple mechanisms. Recent in vitro reports have demonstrated that similar host proteins are involved in facilitating cell entry by SARS-CoV-2, such as ACE2 and TMPRSS2 [5, 24]. Biophysical and structural evidence strongly supports an interaction of ACE2 with SARS-CoV-2 spike protein, similar to SARS-CoV spike protein $[12,13]$. Molecular docking studies have also suggested that SARS-CoV-2 spike protein can interact with cell surface GRP78 [25]. Indirect evidence for a role of CD147 in SARS-CoV-2 binding has been demonstrated in vitro with the use of an anti-CD147 intervention that prevented virus replication [26]. Furthermore, a clinical study with an anti-CD147 intervention reduced symptoms and duration of hospital admission for COVID-19 patients [27]. In summary, although there is evidence that SARS-CoV-2 and SARS-CoV both utilise ACE2 as a receptor to facilitate virus entry, it is possible that differences in host entry mechanisms play a role in the large epidemiological differences between the two viruses, which may include additional unidentified receptors.

ACE2 and TMPRSS2 were identified as cellular entry determinants for SARS-CoV using mechanistic studies. The original report of in situ human lung ACE2 expression described positive immunohistochemical staining for alveoli and airway epithelial cells, and immunocytochemical staining in A549 type II alveolar epithelial cells [28]. ACE2 protein expression is also present in the human lung adenocarcinoma cell line Calu-3 [29]. Similar to ACE2, the original report describing the expression of TMPRSS2 in human respiratory mucosa described expression in airway epithelium and type II alveolar epithelial cells [30]. The specificity of the ACE2 and TMPRSS2 antibodies used for analysis of expression patterns in human lung tissues remains to be addressed.

Affiliations: ${ }^{1}$ Dept of Biology, University of Waterloo, Waterloo, ON, Canada. ${ }^{2}$ Genomics and Regulatory Systems Unit, Okinawa Institute of Science and Technology Graduate University, Onna, Japan. ${ }^{3}$ Faculty of Mathematics, University of Waterloo, Waterloo, ON, Canada. ${ }^{4}$ McMaster Immunology Research Centre, McMaster University, Hamilton, ON, Canada. ${ }^{5}$ Firestone Institute for Respiratory Health - Division of Respirology, Dept of Medicine, McMaster University, Hamilton, ON, Canada. ${ }^{6}$ Michael G. DeGroote Institute for Infectious Disease Research, McMaster University, Hamilton, ON, Canada. ${ }^{7}$ Dept of Biochemistry and Biomedical Sciences, McMaster University, Hamilton, ON, Canada. ${ }^{8}$ Division of Respiratory Medicine, Dept of Medicine, University of British Columbia, Vancouver, BC, Canada. ${ }^{9}$ Nottingham NIHR Biomedical Research Centre, Respiratory Research Unit, University of Nottingham, Nottingham, UK. ${ }^{10}$ Centre for Heart Lung Innovation, University of British Columbia, Vancouver, BC, Canada. ${ }^{11}$ Division of Nephrology, Dept of Medicine, McMaster University, Hamilton, ON, Canada. ${ }^{12}$ A.C. Doxey and J.A. Hirota contributed equally to this article as lead authors and supervised the work.

Correspondence: Jeremy A. Hirota, Firestone Institute for Respiratory Health - Division of Respirology, Dept of Medicine, McMaster University, Hamilton, ON, L8N 4A6, Canada. E-mail: hirotajađmcmaster.ca 
To address the uncertainties related to SARS-CoV-2 receptors in human lung, we performed gene expression and in situ protein profiling of candidate receptors in human airway epithelial cells and lung tissue. Our computational analysis used publicly available microarray gene expression datasets from airway epithelial cells of 515 unique subjects, single cell sequencing data from 10 subjects, and the FANTOM5 dataset for promoter activities of 74 lung-related cell and tissue types. Our proteomic analysis used data from the Human Proteome Map [31] and a dataset from primary human airway epithelial cells grown under air-liquid interface culture conditions [32]. For our in situ protein profiling, we performed immunohistochemical analysis of 98 human lung tissue samples. To determine antibody specificity, we performed immunoblots on protein isolated from Calu-3 cells, primary human airway epithelial cells, primary type II alveolar epithelial cells, the human bronchial epithelium cell (HBEC)-6KT cell line, the A549 type II alveolar epithelial cell line, and HEK cells. Collectively, our data contrast with previous reports, demonstrating rare ACE2 protein expression in the airway epithelium and alveoli of human lung. Our protein expression data are consistent with low ACE2promoter activity in a variety of lung epithelial cell samples and low ACE2 gene expression in both microarray and single cell RNA sequencing (scRNAseq) datasets. We present confirmatory evidence for the presence of TMPRSS2, CD147 and GRP78 protein in vitro in airway epithelial cells and confirm broad in situ protein expression of CD147 and GRP78 in the respiratory mucosa. Our data suggest the presence of a mechanism dynamically regulating ACE2 expression in human lung, perhaps in periods of SARS-CoV-2 infection, and/or that alternate receptors for SARS-CoV-2 exist to facilitate initial host cell infection in lung tissue.

\section{Methods}

Human ethics

Procurement of primary human airway epithelial cells used for immunoblots and lung tissue for immunohistochemistry was approved by the Hamilton (ON, Canada) integrated Research Ethics Board (HiREB 5099T, 5305T, 11-3559 and 13-523-C). The University of British Columbia (Vancouver, BC, Canada) Research Ethics Office approved heart tissue archives and primary human airway epithelial cell collection.

\section{Upper and lower airway gene expression analysis}

Public microarray experiments using Affymetrix chips (HuGene-1.0-st-v1 and HG-U133 Plus 2) on airway epithelial cell samples collected from nasal (GSE19190) or bronchial (GSE11906) brushings of healthy nonsmokers were obtained from the NCBI Gene Expression Omnibus (GEO) database [33, 34]. This resulted in a total of 80 individual samples from the two different experiments that included 11 upper airway samples (nasal $n=11$ ) and 69 lower airway samples (trachea $n=17$, large airway $n=17$, small airway $\mathrm{n}=35$ ). For all dataset samples, raw intensity values and annotation data were downloaded using the GEOquery R package (version 2.52.0) [35] from the Bioconductor project [36]. Probe definition files were downloaded from Bioconductor and probes were annotated using Bioconductor's "annotate" package. All gene expression data were unified into a single dataset that was then normalised by robust multiarray average (RMA) normalisation, and only genes present in both of the Affymetrix platforms $(n=16013)$ were kept for subsequent analyses. Correction of experiment-specific batch effects was performed using the ComBat method [37] implemented using the sva $\mathrm{R}$ package (version 3.32.1) [38]. RMA-normalised expression levels for conventional (ACE2, TMPRSS2, ADAM17 and CTSL (cathepsin L1)) and non-conventional (CD147 and GRP78) SARS-CoV-2 receptor genes were compared across the four defined airway levels, with $C D H 1$ (E-cadherin) expression level included as a positive control with known expression in lung tissue. Gene expression levels were tested for significant differences via pairwise Wilcoxon rank sum tests with Benjamini-Hochberg multiple testing correction using the stats $\mathrm{R}$ package (version 3.6.1). Gene expression box plots were generated with the ggplot2 R package (version 3.2.1).

\section{Analysis of curated bronchial epithelial cell brushing dataset}

A total of 1859 public microarray experiments using Affymetrix chips (HG-U133 Plus 2 and HuGene-1.0-st-v1) on airway epithelial cell samples were selected from the NCBI GEO database. These samples were further filtered by removing individuals with asthma or COPD, resulting in a total of 504 individual healthy samples (GSE4302, 28 samples; GSE67472, 43 samples; GSE37147, 159 samples; GSE108134, 274 samples). Within this dataset, sex and/or age information was included for 310 samples; of these, sex data were available for 86 females and 106 males. Smoking status information was also provided for 451 samples, with 260 current smokers, 82 former smokers and 109 never-smokers.

For all dataset samples, raw intensity values and annotation data were downloaded as described. Probe definition files were retrieved as described. All gene expression data were unified into a single dataset that was then RMA-normalised, and only genes present in both of the Affymetrix platforms $(n=16105)$ were kept for subsequent analyses. Correction of experiment-specific batch effects was performed as described. 
Analysis of promoter activity from the FANTOM5 dataset

The FANTOM5 promoterome dataset [39] for the hg38 assembly [40] was used to examine promoter activity of SARS-CoV-2-related human genes, namely ACE2, TMPRSS2, ADAM17, CTSL, CD147 and GRP78. Using the ZENBU genome browser [41], the nearest cap analysis of gene expression (CAGE) peak upstream and on the same strand as each of the aforementioned genes was extracted and analysed. The dataset consists of CAGE promoter activity data for 1866 primary cells, cell lines and tissues from humans, and is quantified as normalised transcripts per million (TPM). A subset of FANTOM5 CAGE data (120 samples) is presented, considering only samples related to lung, gut, heart and prostate tissues (consisting of 74, 19, 15 and 12 samples, respectively). Normalised TPM values for each CAGE peak, an approximation for promoter activity, were $\log _{10}$ transformed and separated according to tissue and cell type, and the radius of each point is proportional to these transformed normalised TPM values.

\section{Analysis of protein abundance from proteomic datasets}

Publicly available human proteomic data from the datasets of KIM et al. [31] and FosTER et al. [32] were used to evaluate SARS-CoV-2 receptor-related protein expression in different human tissues and experimental conditions. Expression values were extracted from the dataset of KIM et al. [31] for ACE2, TMPRSS2, ADAM17, CTSL, CD147 and GRP78, using CDH1 as a control for airway cells. Data were created using the pheatmap package in $\mathrm{R}$ (version 1.0.12) and expressed as $\log _{10}$-transformed to facilitate visualisation. Proteomic data from the dataset of Foster et al. [32] consist of bronchial epithelial cells collected from healthy nonsmokers $(n=4$; males) and exposed to PBS control vehicle. Intensity values for ACE2, TMPRSS2, ADAM17, CD147 and GRP78 were extracted, with CDH1 included as a positive control. Intensity values were determined by the original study authors via normalisation of all detected peptide intensities associated with a given parent protein [32]. Box plots were generated with the ggplot2 $\mathrm{R}$ package (version 3.2.1) with intensity values $\log _{10}$-transformed for visualisation purposes.

\section{Analysis of single cell RNA sequencing data}

Data preprocessed using the Cell Ranger pipeline (10x Genomics) were obtained from GSE135893. Samples from 10 control subjects and 12 idiopathic pulmonary fibrosis patients were downloaded and post-processed with the Seurat package in $\mathrm{R}$ [42]. Cell populations were defined using the markers provided in the source paper [43]. Cells belonging to the 10 control subjects were used for further analysis. Visualisations of violin plots were created using Seurat.

\section{Primary human airway epithelial cells}

The human lung adenocarcinoma cell line, Calu-3, was grown under culture conditions defined by the supplier (ATCC HTB-55). Primary human airway epithelial cells isolated via bronchial brushings from consented healthy individuals were grown in PneumaCult ExPlus (Stemcell Technologies, Vancouver, BC, Canada) under submerged monolayer culture conditions and used between passages 1 and 4 . The human bronchial epithelial cell line, HBEC-6KT, was grown under submerged monolayer culture conditions in keratinocyte serum-free media supplemented with epidermal growth factor $\left(0.4 \mathrm{ng} \cdot \mathrm{mL}^{-1}\right)$ and bovine pituitary extract $\left(50 \mu \mathrm{g} \cdot \mathrm{mL}^{-1}\right)[44-47]$.

\section{Immunoblots}

Cell protein was isolated using RIPA lysis buffer (VWR, Mississauga, ON, Canada) supplemented with protease inhibitor cocktail (Sigma, Oakville, ON, Canada) with quantification performed using Bradford assay reagents (Bio-Rad, Mississauga, ON, Canada). Immunoblots were performed using stain-free $4-20 \%$ pre-cast gradient gels and imaged on a ChemiDoc XRS+ Imaging system (Bio-Rad). For each immunoblot, $20 \mu \mathrm{g}$ of protein was added per lane. ACE2 (MAB933, monoclonal, clone 171606, $2 \mu \mathrm{g} \cdot \mathrm{mL}^{-1}(\mathrm{R} \& \mathrm{D}$ Systems)), TMPRSS2 (HPA035787, polyclonal, $0.4 \mu \mathrm{g} \cdot \mathrm{mL}^{-1}$ (Atlas Antibodies)), CD147 (ab666, monoclonal, clone MEM-M6/1, $1 \mu \mathrm{g} \cdot \mathrm{mL}^{-1}$ (Abcam)) and GRP78 (610979, monoclonal, clone 40/BiP, $0.25 \mu \mathrm{g} \cdot \mathrm{mL}^{-1}$ (BD Biosciences), and HPA038845, rabbit polyclonal (Atlas Antibodies)) primary antibodies were diluted in $5 \%$ skimmed milk/TBS with $0.1 \%$ Tween- 20 and incubated overnight on a rocker at $4^{\circ} \mathrm{C}$ with detection performed the following day using an anti-mouse (ACE2, CD147 and GRP78 (BD Biosciences)) or anti-rabbit (TMPRSS2 and GRP78 (Atlas Antibodies)) horseradish peroxidase (HRP)-conjugated secondary antibodies at 1:3000 for $2 \mathrm{~h}$ at room temperature (Cell Signaling, Danvers, MA, USA). Visualisation of TMPRSS2, CD147 and GRP78 was performed using Clarity Western enhanced chemiluminescence (ECL) Substrate, while ACE2 was visualised with Clarity Max ECL Substrate (Bio-Rad). Total protein loading images were collected as a qualitative visualisation of protein loading between sample types [48]. The immunogen for ACE2 primary antibody is mouse myeloma cell line NS0-derived recombinant human ACE2 Gln18-Ser740 (predicted). The immunogen for TMPRSS2 primary antibody is the recombinant protein epitope signature tag antigen sequence, GSPPAIGP YYENHGYQPENPYPAQPTVVPTVYEVHPAQYYPSPVPQYAPRVLTQASNPVVCTQPKSPSGTVCTSKT. 
The immunogen for the CD147 primary antibody is recombinant full-length protein corresponding to human CD147. The immunogen for the GRP78 BD Biosciences primary antibody is human BiP/GRP78 amino acids 525-628. The immunogen for the GRP78 Atlas Antibodies primary antibody is the recombinant protein epitope signature tag antigen sequence, EKFAEEDKKLKERIDTRNELESYAYSLKNQI GDKEKLGGKLSSEDKETMEKAVEEKIEWLESHQDADIEDFKAKKKELEEIVQPIISKL.

Independent immunoblot analysis (L. Organ, C. Joseph, A. John and G. Jenkins) was performed on A549, HEK and immortalised human bronchial epithelial cells. Equal amounts of protein $(20 \mu \mathrm{g})$ were loaded on to $4-12 \%$, Bis-Tris gradient gels (NP0326BOX; ThermoFisher) with anti-ACE2 (ab108252, rabbit monoclonal, clone EPR4435(2), 1/500 dilution of stock antibody; Abcam). A loading control of GAPDH was used to demonstrate protein loading (ab181603, rabbit monoclonal, EPR16884, 1/10000 dilution of stock antibody; Abcam). Visualisation was performed with ECL Clarity (Bio-Rad) on a Licor C-DiGit.

\section{Immunohistochemistry}

Formalin-fixed paraffin-embedded human lung tissue from non-diseased regions was obtained from archived tissue blocks from patients who had undergone lung resection for clinical care. Human heart tissue was from the University of British Columbia Cardiovascular Tissue Registry. Sections $4 \mu \mathrm{m}$ thick were cut and stained for ACE2 $\left(15 \mu \mathrm{g} \cdot \mathrm{mL}^{-1}\right)$, TMPRSS2 $\left(10 \mu \mathrm{g} \cdot \mathrm{mL}^{-1}\right), \mathrm{CD} 147\left(5 \mu \mathrm{g} \cdot \mathrm{mL}^{-1}\right)$, and GRP78 (HPA038845, 1/200 dilution) using the same antibodies used for immunoblot analysis. All staining was performed on a Leica Bond RX system with Leica Bond reagents, heat-induced antigen retrieval at $\mathrm{pH} 6$ (20 min) with primary antibody incubation for $20 \mathrm{~min}$. Digital slide scanning was performed using an Olympus VS120-L100 Virtual Slide System at 40× magnification with VS-ASW-L100 V2.9 software and a VC50 colour camera, followed by image visualisation with HALO image analysis software.

\section{Results}

Candidate genes important in SARS-CoV-2 infection are detectable at varying levels in human airway epithelial cells and lung tissue

We performed a targeted analysis of ACE2, TMPRSS2, ADAM17, CTSL, CD147 and GRP78 gene expression as candidates important for SARS-CoV-2 infection in human airway epithelial cells. Here and throughout the gene expression analyses, $\mathrm{CDH1}$ (E-cadherin) was used as a control for lung epithelial cell phenotype. We first examined these genes in a curated dataset of upper and lower airway epithelial cell gene expression from the nasal sinus to the 12th generation of airway in the lung (figure 1).

In the upper airways, all candidates were expressed, with the highest level observed for GRP78 and the lowest level observed for ACE2. Analysis along multiple generations of the lower airways (trachea, large (4th-6th generation) and small airways (10th-12th generation)) revealed identical relative expression patterns, with ACE2 being the least expressed and GRP78 being the highest expressed. ACE2 gene expression showed the greatest variability along the upper and lower airways, with greatest expression observed in the trachea samples and the lowest expression in the small airway (figure 1).

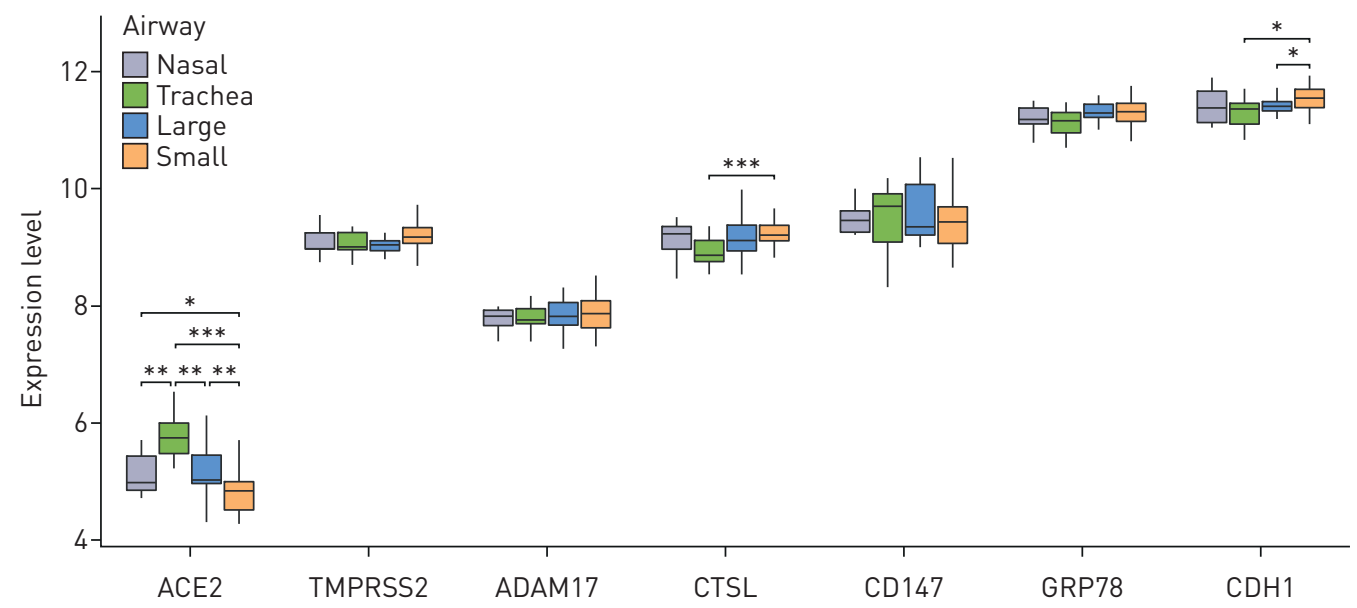

FIGURE 1 Microarray expression profiles of candidate SARS-CoV-2 receptor genes in upper and lower airways. Normalised $\log _{2}$ expression levels for ACE2 langiotensin-converting enzyme 2), TMPRSS2, ADAM17, CTSL (cathepsin L1), CD147 and GRP78 genes compared across the upper airway (nasal) and lower airways (trachea, large airway and small airway). $C D H 1$ (E-cadherin) gene expression level is included as a positive control. Statistical values for comparisons for each gene at each airway generation were calculated; those not shown were nonsignificant. *: $p<0.05 ;{ }^{* *}: p<0.01 ;{ }^{* * *}: p<0.001$. 
Following our observation of consistent expression along the upper and lower airways of candidate genes important in SARS-CoV-2 infection, we determined whether sex or age affected gene expression levels in healthy individuals using a curated dataset of bronchial brushings from 504 healthy subjects (supplementary table S1). The expression levels for the candidate genes in healthy subjects paralleled the patterns observed in the smaller survey of upper airways, trachea, large and small airways (figure 2).

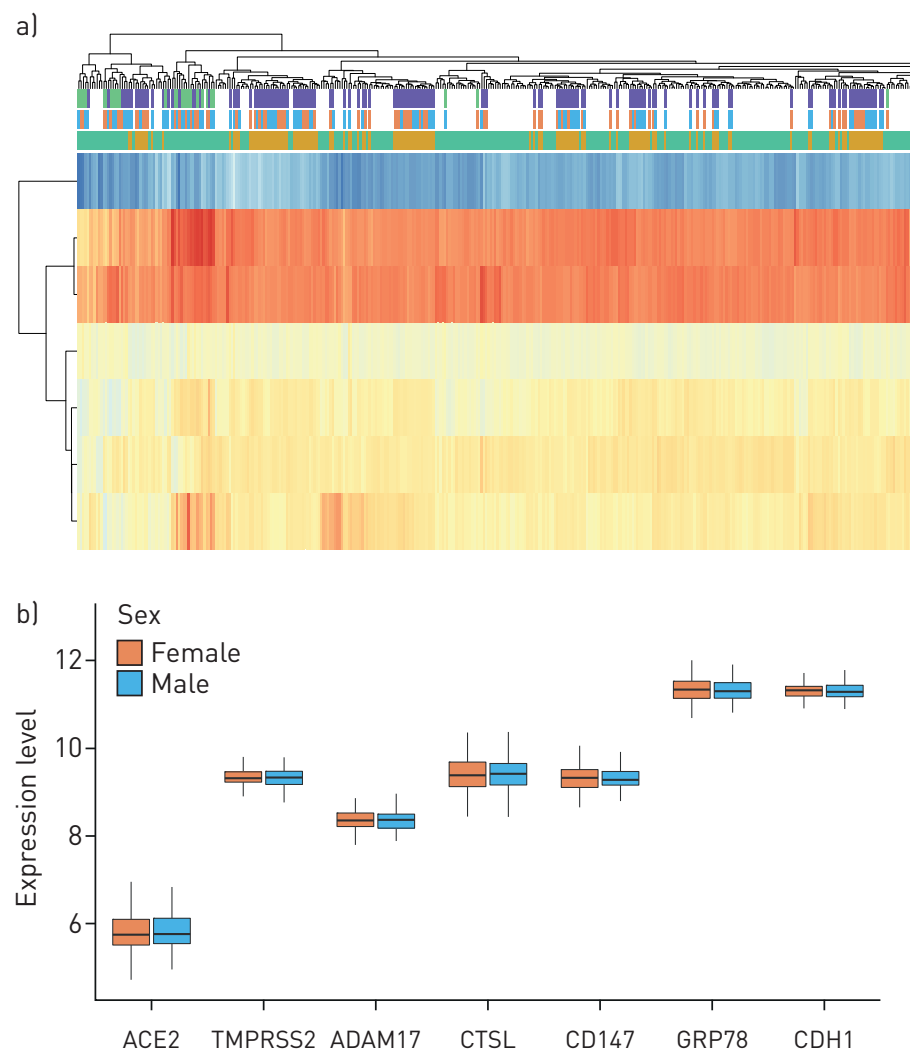

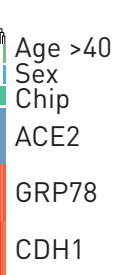

ADAM17

CD147

TMPRSS2

CTSL

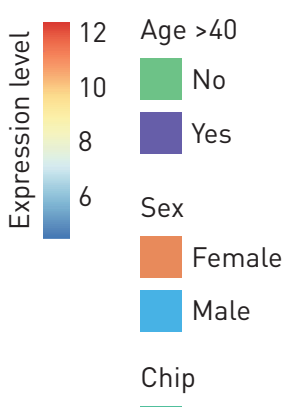

HG-U133 Plus 2

HuGene-1.0-st-v1
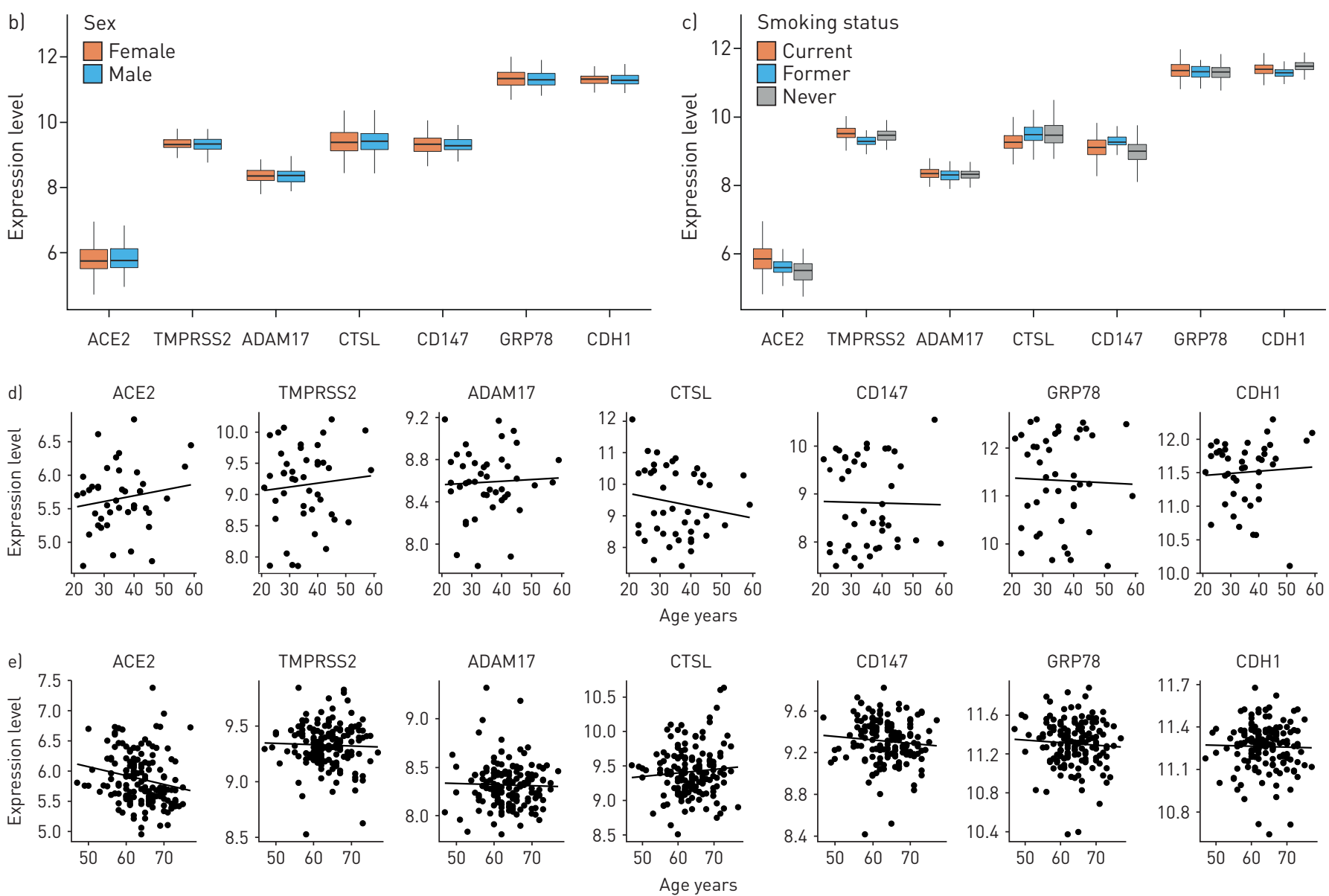

FIGURE 2 Microarray expression profiles of candidate SARS-CoV-2 receptor genes in lower airway epithelial cells, analysed by age and sex. al Clustered heatmap of $\log _{2}$ expression levels from NCBI Gene Expression Omnibus (GEO) samples ( $\mathrm{n}=504$ ), annotated by age, sex and microarray chip platform. Expression values reflect signal intensities, indicating lowest detected expression of ACE2 (angiotensin-converting enzyme 2) and highest expression of GRP78 and CDH1 (E-cadherin). b and c) Box plots of expression levels separated by b) sex (n=194) and c) smoking status ( $n=451)$. $d$ and e) Plots of gene expression levels versus age, with linear regression lines of best fit, for datasets that used either d) the HG-U133 Plus 2 microarray $(n=43)$ or e) the HuGene-1.0-st-v1 microarray $(n=181)$. Correlations were performed separately between platforms because of differences in their age distributions. e) A weak negative correlation ( $r=-0.20, p=0.015)$ was detected for ACE2 in the dataset that used the HuGene-1.0-st-v1 microarray. 
Median ACE2 gene expression was the lowest, while GRP78 gene expression was the highest (figure 2a). No gene candidate demonstrated sex dependence for expression levels (figure 2b). ACE2, TMPRSS2, CD147 and GRP78 were elevated in current smokers relative to never-smokers (figure 2c and supplementary table S2). CTSL was reduced in current smokers relative to never-smokers. No microarray chip-dependent effects were observed for relationships between sex or smoking status and gene expression. For quantitative analyses related to age and gene expression, our curated database was divided into datasets that used either the HG-U133 Plus 2 or HuGene-1.0-st-v1 microarray due to differences in age distributions. In the HuGene-1.0-st-v1 dataset $(\mathrm{n}=181)$, which included a greater proportion of older individuals ( $>50$ years), we observed reduced ACE2 gene expression with age (figure $2 \mathrm{e} ; \mathrm{p}<0.05$ ).

Promoter activity data of each of the candidate genes important in SARS-CoV-2 binding and infection were extracted and analysed from the FANTOM5 dataset, which includes 1866 primary cells, cell lines, and tissue sample types (figure 3). We selected all sample formats that included "lung", "nasal", "airway" or "olfactory", to identify lung-specific sample types. Gut, heart and prostate tissue samples were analysed as controls. Consistent with our observed gene expression analysis along the upper and lower airways, normalised TPM values for each CAGE peak demonstrated that CD147 promoter activity was elevated relative to ACE2 promoter activity across airway epithelial cells and lung tissue samples. CTSL promoter activity was the lowest of all candidate genes, which contrasted with the modest expression observed at the gene level (figure 2a). Both microarray gene expression analysis and promoter activity were consistent with results of candidate gene expression in a scRNAseq dataset of 10 healthy subjects (supplementary figure S1).

Collectively, our gene expression analysis of the upper and lower airways of healthy males and females of diverse ages suggests that ACE2 gene expression is low relative to all other candidate SARS-CoV-2 receptor genes analysed in human airway epithelial cells. Furthermore, we observe no sex-dependent or age-dependent expression patterns of any candidates at the gene level, although smoking status did have an impact on gene expression levels.

In vitro and in situ protein profiling reveals distinct expression patterns for candidates important in SARS-CoV-2 infection

Analysis of transcriptional data may not be indicative of in situ protein expression levels [49]. To extend our gene expression observations, we mined publicly available proteomic data from whole lung and primary human airway epithelial cell cultures and performed in vitro immunoblots on human airway epithelial cell lysates and in situ protein immunohistochemistry on human lung tissue using the same antibodies for each method.

The Human Proteome Map is a publicly available resource that includes select adult and fetal tissues and circulating immune cell populations [31]. Using this resource, we examined protein expression of ACE2, TMPRSS2, ADAM17, CTSL, CD147 and GRP78. In human lung tissue homogenate, ACE2 was not detected, while being detected in heart, gut and testes, known positive control tissues (figure 4a). The rank order of the remaining molecules in human lung tissue homogenate was: GRP78>CD147 $>$ CTSL $>$ ADAM17>TMPRSS2. Human lung tissue homogenate is a heterogeneous population of cells, precluding the ability to associate protein expression to a given cell type. We therefore interrogated a publicly available proteomic dataset derived from primary human airway epithelial cells grown under air-liquid interface culture conditions [32], examining the same candidates. Again, ACE2 protein expression was not detectable (figure 4b). CD147, GRP78 and CTSL were expressed with multiple peptide counts, while TMPRSS2 and ADAM17 were only marginally expressed with low peptide counts. Collectively, two proteomic datasets from distinct lung sample formats provide complementary and consistent expression profiles of candidate molecules important in SARS-CoV-2 infection.

To localise the in situ expression of the candidate molecules of interest at the protein level, we pursued immunohistochemical analysis paired with immunoblot validation of the specificity of the selected antibodies for recognition of proteins of the predicted molecular weight. An anti-ACE2 antibody detected only a single band in Calu-3 cells at the predicted molecular weight of ACE2 protein ( 110 kDa) (figure 5a, lanes 1-3). The anti-ACE2 antibody required the use of a super-sensitive ECL solution. No ACE2 protein was detected in primary airway epithelial cells or the HBEC-6KT cell line, despite confirmation of protein loading (figure 5a, lanes 4-9, protein loading shown underneath main blot). Independent immunoblotting with a distinct anti-ACE2 primary antibody was performed, with a single band observed in HEK cells, but not in immortalised human bronchial epithelial cells or A549 cells (supplementary figure S2).

An anti-TMPRSS2 antibody detected multiple bands in all airway epithelial cell samples, with a dominant band at the predicted molecular weight of $\sim 57 \mathrm{kDa}$ (figure $5 \mathrm{~b}$ ). These patterns were conserved across all cell types that were analysed. 

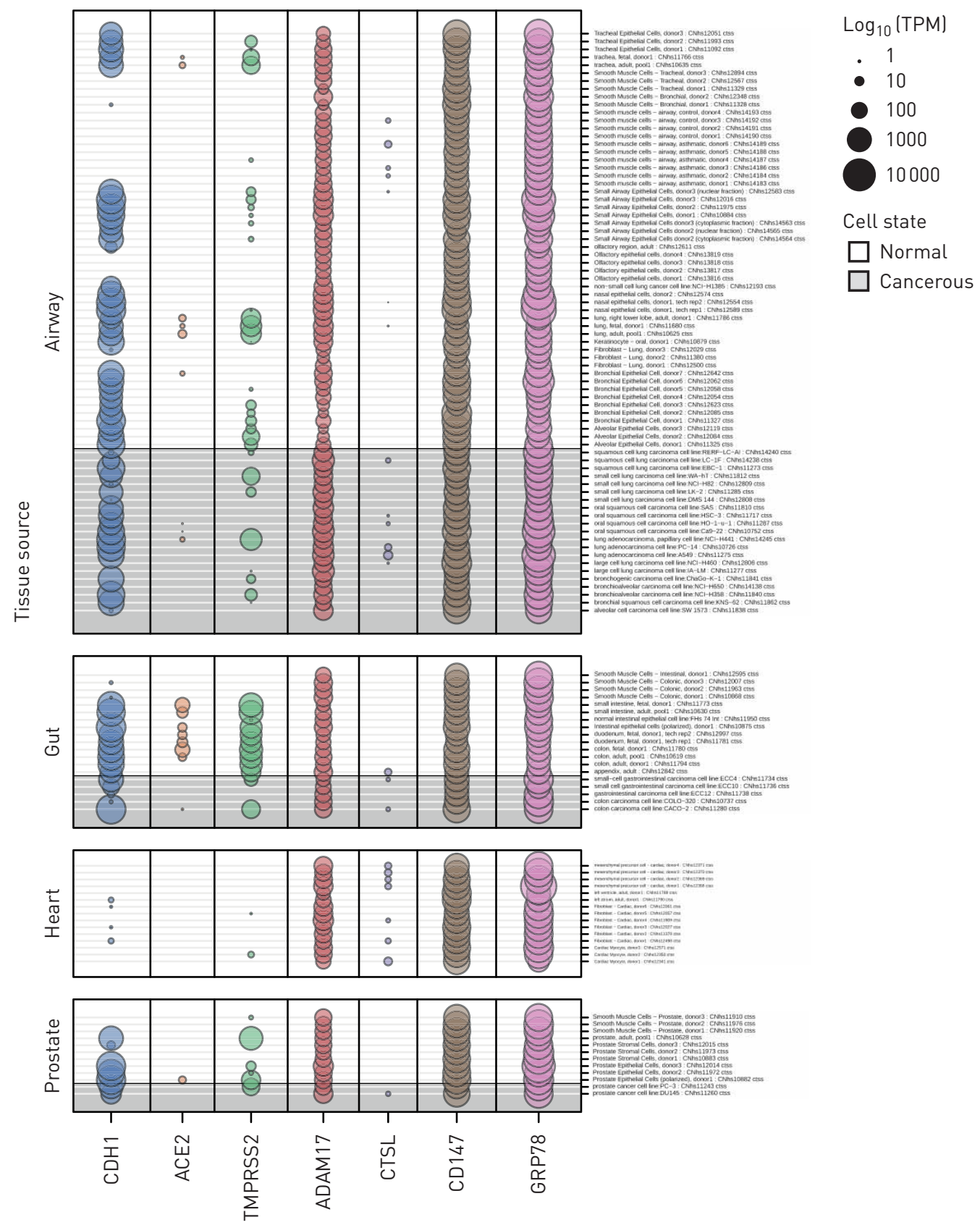

FIGURE 3 Promoter activity for SARS-CoV-2-related genes from the FANTOM5 cap analysis of gene expression (CAGE) dataset. The FANTOM5 CAGE data consist of quantified promoter expression levels across the human genome for 1866 samples from primary cells, cell lines and tissue samples. The FANTOM5 CAGE promoter activity data for several SARS-CoV-2-related genes are shown for samples related to lung, gut, heart and prostate tissues $(n=120)$. Dot sizes are proportional to promoter activity, depicted as $\log _{10}$-transformed normalised transcripts per million (TPM). Notably, angiotensin-converting enzyme 2 (ACE2) is either not expressed or expressed at low levels $(<1$ TPM in all but one sample) in the airway, including measurements from healthy and cancerous cells.

An anti-CD147 antibody detected a single band in all airway epithelial cell samples, with a dominant band at the predicted molecular weight of $\sim 55 \mathrm{kDa}$ (figure $5 \mathrm{c}$ ). The immunoblot bands were consistent with the heavy glycosylation of CD147 [50].

An anti-GRP78 antibody (BD Biosciences 610979) detected a single band in all airway epithelial cell samples, with a dominant band at the predicted molecular weight of $\sim 78 \mathrm{kDa}$ (figure $5 \mathrm{~d}$ ).

The immunoblots using anti-ACE2, anti-CD147 and anti-GRP78 demonstrated a single band of predicted molecular weight, suggesting that observed immunohistochemical staining should be specific to the protein 
a)

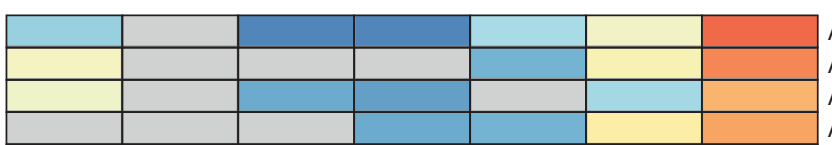

Adult adrenal

Adult colon

$\log _{10}$-transformed

Adult oesophagus

Adult frontal cortex

Adult gallbladder

Adult heart

Adult kidney

Adult liver

Adult lung

Adult ovary

Adult pancreas

protein expression

Adult prostate

Adult rectum

Adult retina

Adult spinal cord

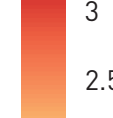

2.5

Adult testis

Adult urinary bladder

B-cells

CD4 cells

CD8 cells

Monocytes

NK cells

Placenta

Platelets

Fetal brain

Fetal gut

Fetal heart

Fetal liver

Fetal ovary

Fetal testis

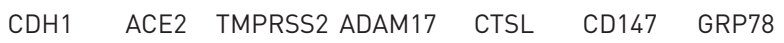

b)

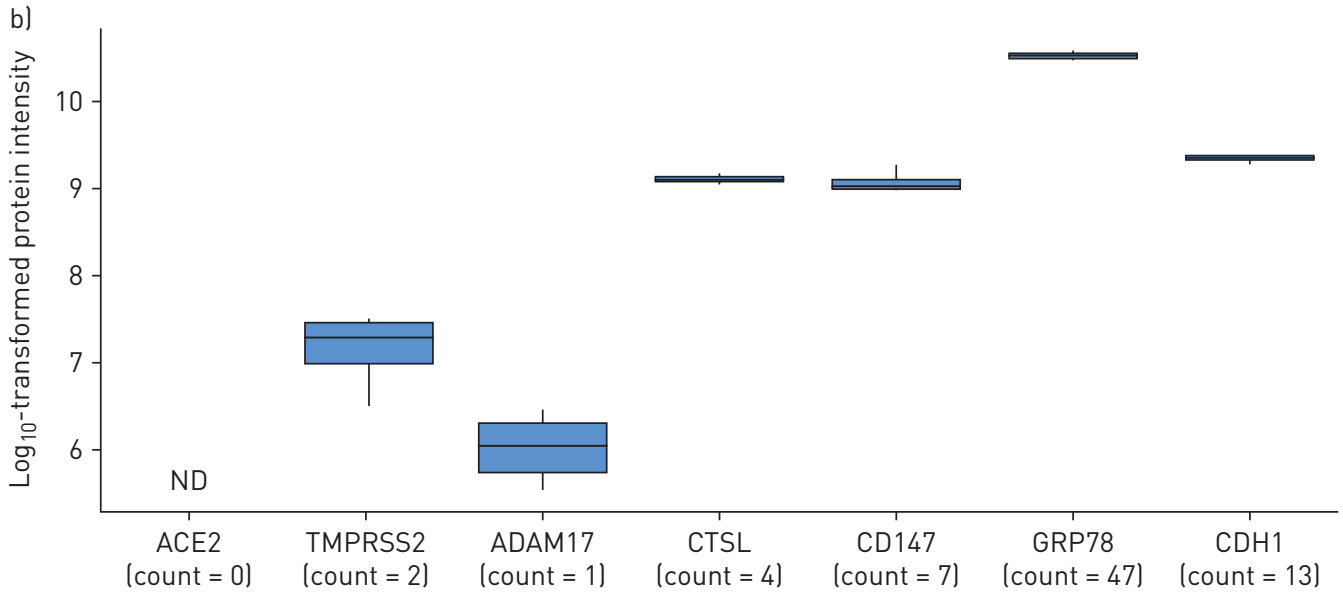

FIGURE 4 Proteomic profiles of candidate SARS-CoV-2 receptor genes in human tissue and airway epithelial cells. a) Intensity values of protein expression from KIM et al. [31] for the genes ACE2 langiotensin-converting enzyme 2), TMPRSS2, ADAM17, CTSL (cathepsin L1), CD147 and GRP78. CDH1 (E-cadherin) intensity is included as a positive control for expression in airway cells. Intensity values have been $\log _{10}$-transformed to facilitate comparison between candidates with different basal expression levels across tissue types. Grey cells in the heatmap correspond to an untransformed intensity of 0 and represent an undetectable signal. b) Intensity values $\log _{10}$-transformed for visualisation of ACE2, TMPRSS2, ADAM17, CTSL, CD147 and GRP78 proteins in human airway epithelial cells from healthy nonsmokers (n=4; males) grown under air-liquid interface culture conditions [32]. CDH1 intensity is included as a positive control. Counts indicating the number of detected peptides associated with each parent protein are provided. ND: the protein was not detected in this study.

of interest based on the target epitope, as both methods detect denatured proteins [51]. The same anti-ACE2 and anti-CD147 antibodies were validated for immunohistochemistry. The anti-TMPRSS2 was used for immunohistochemistry, although the multiple bands observed by immunoblot caution the specificity of any observed in situ staining. Attempts to optimise anti-GRP78 antibody application for immunohistochemistry were unsuccessful, requiring additional antibody interrogation with HPA038845 
a)

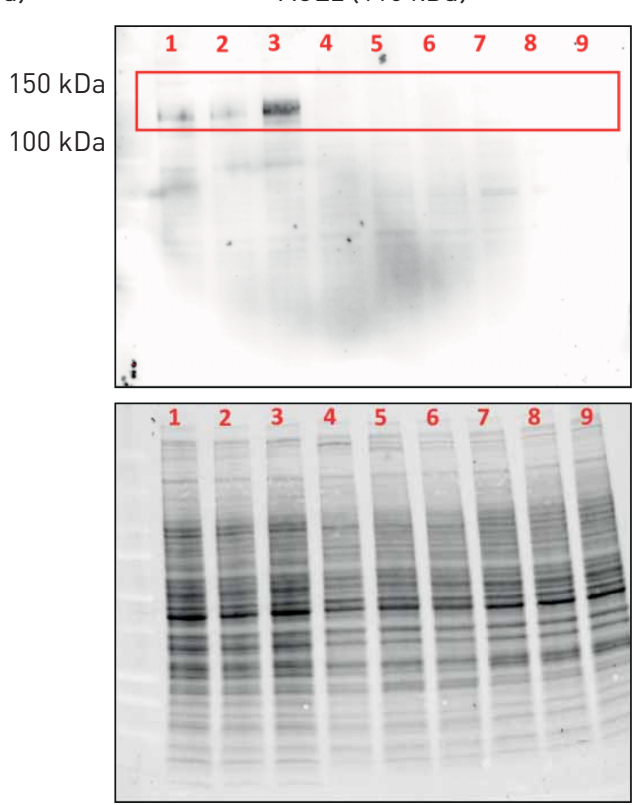

c)
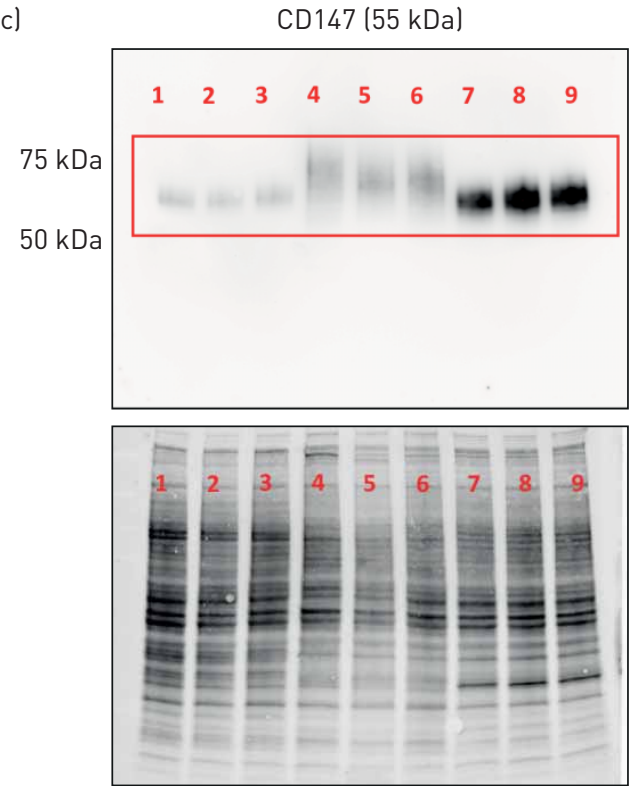

b)
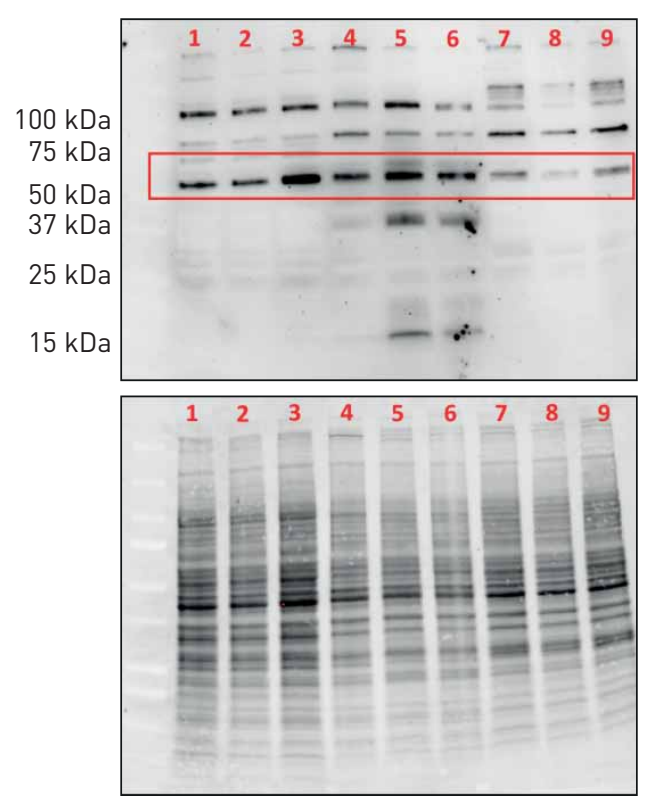

d)

GRP78 (78 kDa)
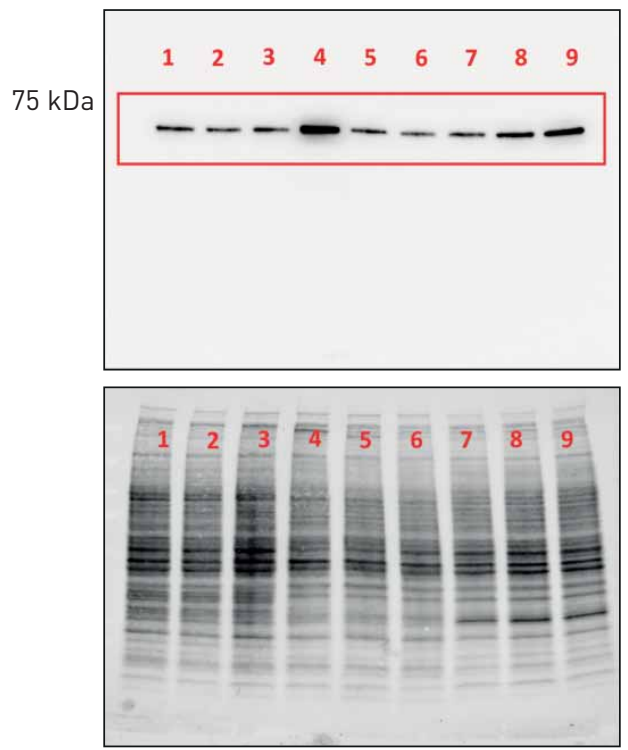

FIGURE 5 Immunoblot analysis of ACE2 (angiotensin-converting enzyme 2), TMPRSS2, CD147 and GRP78 protein expression in human airway epithelial cell protein lysates. a) ACE2 with single band for predicted molecular weight of $110 \mathrm{kDa}$ (red box). b) TMPRSS2 with multiple bands including a dominant band at predicted molecular weight of $57 \mathrm{kDa}$ (red box). c) CD147 with a single broad band around predicted molecular weight of $55 \mathrm{kDa}$ (red box). d) GRP78 with a single band at predicted molecular weight of $78 \mathrm{kDa}$ (red box). Lanes 1-3: Calu-3 cells. Lanes 4-6: primary human airway epithelial cells. Lanes 7-9: human bronchial epithelium cell (HBEC)-6KT cell line. All cells grown under submerged monolayer conditions, with $\mathrm{n}=3$ independent passages (Calu-3 or HBEC-6KT) or donor samples (primary human airway epithelial cells; nonsmoker, healthy subjects). For each independent blot of each protein, all of the same samples were run. A total protein loading control is provided to demonstrate protein loaded for each sample.

(Atlas Antibodies), which was suitable for both immunoblotting and immunohistochemistry (supplementary figure S3).

ACE2 immunohistochemistry revealed only select staining in rare cells in the airways and the alveoli of all 98 human lung samples analysed, which included healthy subjects and those with chronic lung diseases (figure 6). A single healthy human sample contained one positive airway epithelial cell with additional positive staining in the peripheral lung in cells with type II alveolar epithelial cell morphology (figure 6a). 
A representative image of a sample from a smoker with COPD (figure 6b) shows no ACE2 protein staining in the airway epithelium and a rare positive cell in sub-basement membrane tissue. Quantification of positive pixel count for ACE2 staining normalised to total tissue pixel count revealed no differences between healthy nonsmokers and tobacco smokers (supplementary figure S4). Lung microvasculature and human heart tissue had positive staining (supplementary figures S5 and S6), consistent with previously described reports for ACE2 protein staining patterns $[52,53]$.

TMPRSS2 immunohistochemistry revealed diffuse staining in the airway epithelium and in immune cells in the lung periphery, with greater staining in smokers with COPD (figure 6). These observations were consistent in the majority of the 98 human samples examined.

CD147 immunohistochemistry revealed strong membrane-restricted staining in the airway epithelium and diffuse staining in immune cells in the lung periphery (figure 6). CD147 displayed greater staining in smokers with COPD. These observations were consistent in the majority of the 98 human samples examined.

GRP78 immunohistochemistry revealed diffuse staining in airway and alveolar epithelium and in immune cells in the lung periphery (figure 6; 49 samples). No qualitative differences in GRP78 staining were observed between healthy subjects and smokers with COPD.

Collectively, our in vitro and in situ protein profiling is consistent with our gene expression analysis, with CD147 and GRP78 protein expression dominant over TMPRSS2 and ACE2. Additional examples of staining in lung tissue are provided in supplementary figure S7. ACE2 protein expression is rare in human lung tissue and found in select cells in both healthy individuals and those with chronic lung diseases.
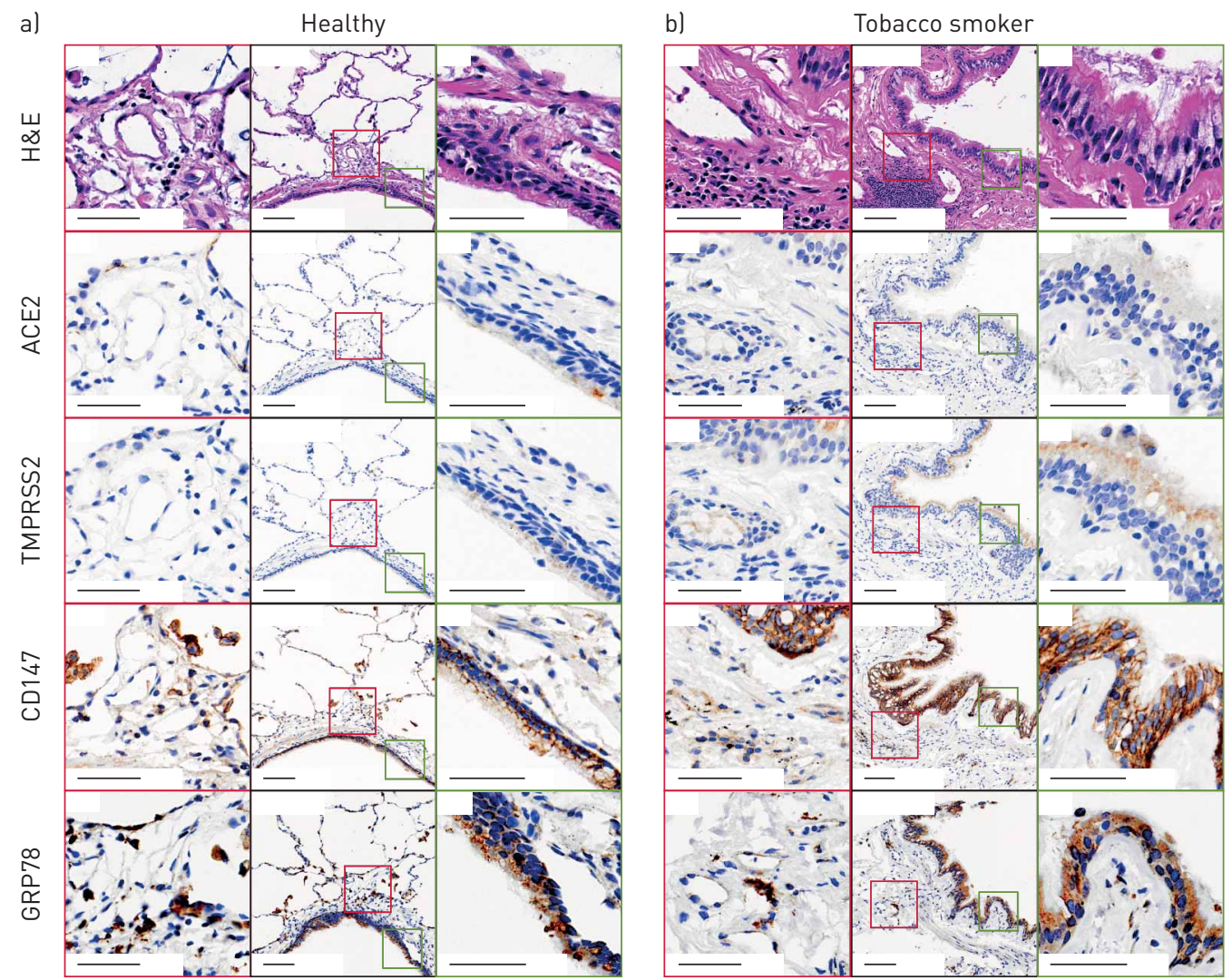

FIGURE 6 Immunohistochemical localisation of ACE2 langiotensin-converting enzyme 2), TMPRSS2, CD147 and GRP78 protein in human lung tissue. Representative samples from al a healthy nonsmoker with no underlying chronic airway disease, and b) a smoker with COPD. Black outlines: low magnification (12x) of conducting airways with airway epithelium; scale bars $100 \mu \mathrm{m}$. Green outlines: high magnification regions $(60 \times)$ of conducting airway epithelium that are defined in the low magnification images by green squares; scale bars $50 \mu \mathrm{m}$. Red outlines: high magnification regions $(50 \times)$ of lung tissue away from the airway lumen that are defined in the low magnification images by red squares; scale bars $50 \mu \mathrm{m}$. H\&E: haematoxylin and eosin. Positive immunohistochemical staining is rust/brown. Total number of independent samples analysed was 49-98. 
TMPRSS2 and CD147 protein expression are potentiated in individuals with a history of tobacco smoking and a diagnosis of COPD.

\section{Discussion}

The global COVID-19 pandemic that emerged in late 2019 is caused by SARS-CoV-2. The possible host receptor(s) for SARS-CoV-2 have not been exhaustively surveyed in human lung tissue at the gene and protein level. Understanding the expression levels and localisation of candidate SARS-CoV-2 receptors in host lung tissue may provide insights into therapeutic interventions that might reduce disease spread, viral replication or disease pathology. To address this knowledge gap, we performed gene expression, proteomic profiling at the tissue and cell level, and in situ protein profiling of candidate receptors in human airway epithelial cells and lung tissue (summarised in figure 7). Collectively, our data demonstrate rare ACE2 protein expression in human airway epithelial cells in vitro and in situ. Our protein expression data are consistent with low ACE2 promoter activity in a panel of lung epithelial cell samples and low ACE2 gene expression in bronchial epithelial cells (microarray) and lung cells (scRNAseq). We present confirmatory evidence for the presence of TMPRSS2, CD147 and GRP78 protein in vitro in airway epithelial cells and confirm broad in situ protein expression of CD147 and GRP78 in the respiratory mucosa. Our data suggest that for ACE2 to be an integral receptor for SARS-CoV-2, mechanisms are likely to exist that dynamically regulate expression in human lung, perhaps in periods of SARS-CoV-2 infection [54]. It is also possible that alternate receptors for SARS-CoV-2 are important in initial host cell infection.

Using a curated microarray gene expression dataset generated from bronchial brushings of 504 healthy subjects that considers the limitations of merging multiple datasets from distinct sources, we observed that sex did not correlate with gene expression of any candidate host molecule involved in SARS-CoV-2

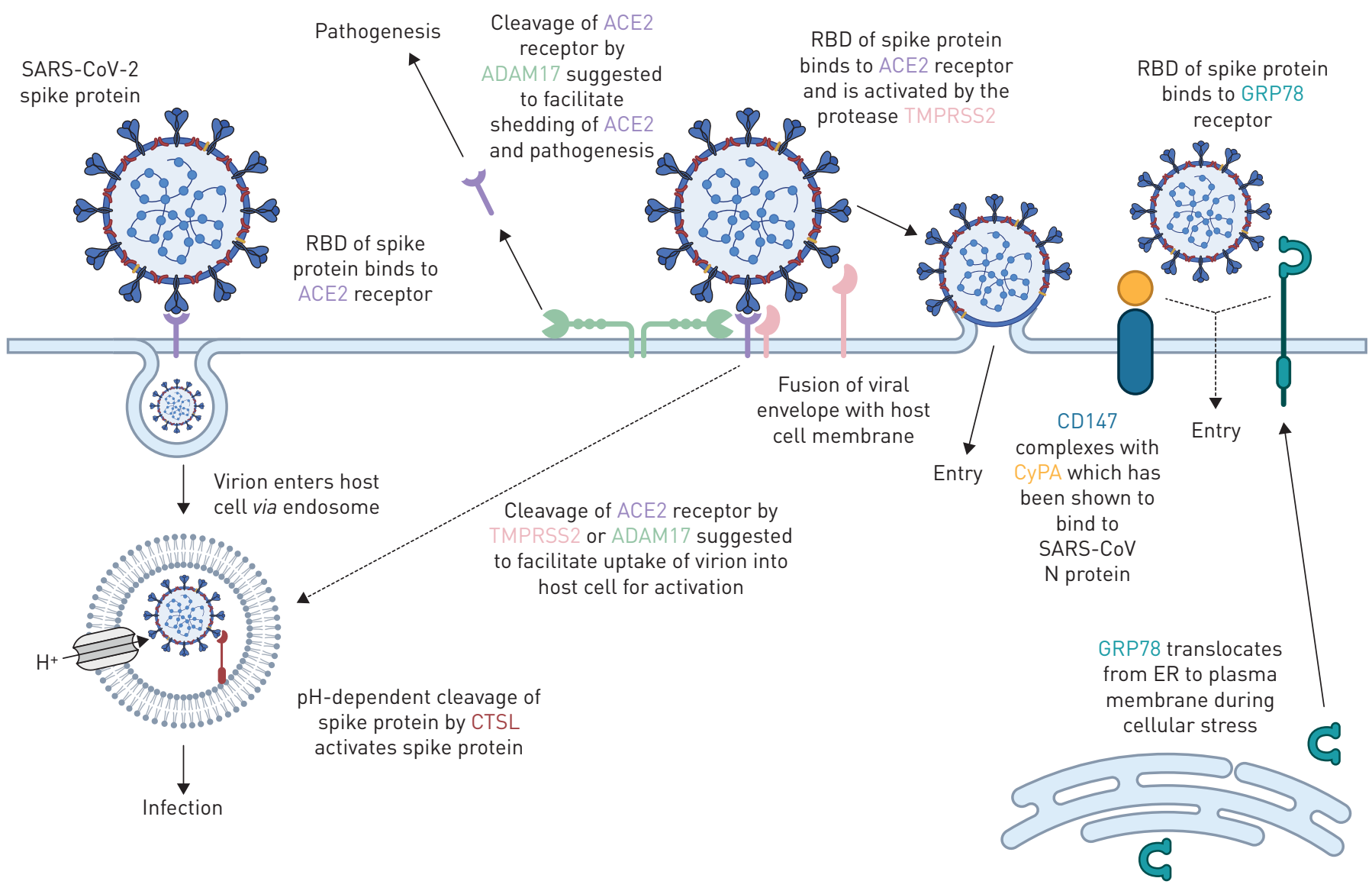

FIGURE 7 Proposed functions of host airway epithelial cell molecules for interaction with SARS-CoV-2. Proteins associated lor suggested to be associated) with host cell entry of SARS-CoV-2 and the activation of the SARS-CoV-2 spike protein (SARS-S) are displayed. Angiotensin-converting enzyme 2 (ACE2) is suggested as the primary SARS-S receptor for viral entry (interaction of ACE2 with SARS-S receptor-binding domain (RBD) leading to endosomal viral uptake), followed by activation of SARS-S via pH-dependent cleavage mediated by cathepsin L1 (CTSL). Secondary methods of viral entry and SARS-S activation are likely to involve proteases (e.g. TMPRSS2 and ADAM17) and/or secondary receptors (CD147 and GRP78). Dashed lines indicate mechanisms that have not been fully validated. CyPA: cyclophilin A; N protein: nucleocapsid protein; ER: endoplasmic reticulum. Adapted from [20] with updates and additional information on candidate host molecules. Figure created with BioRender.com. 
infection and that ACE2 and TMPRSS2 were the lowest expressed genes of interest examined. In one dataset, ACE2 gene expression modestly decreased with age, although protein level confirmation was not possible. The low level of ACE2 and TMPRSS2 gene expression in bulk bronchial epithelial cell gene expression samples suggests low levels of cells expressing both of these genes within this lung tissue. We confirm that tobacco smoking is associated with elevated ACE2 gene expression levels in bronchial epithelial cell samples [55], although we were unable to confirm this with immunohistochemistry analysis of protein on human lung samples.

Advances in transcriptomics have enabled scRNAseq that has identified unique and rare cell types in human lung that may have importance in health and disease $[56,57]$. scRNAseq provides an opportunity to look at transcriptional profiles in subsets of cell populations, which may isolate a cell signal from a bulk sample. We therefore utilised scRNAseq data from healthy human lung samples as a parallel approach. The resolution of scRNAseq for subpopulations of epithelial cells revealed low or absent expression of ACE2 gene in all populations examined, whereas CD147 and GRP78 were present in all populations. Our results are consistent with current publicly available data that discuss the presence of rare ACE2/ TMPRSS2-positive cells [54]. Using lung samples from eight individuals (four HIV and active tuberculosis double positive, two HIV positive and tuberculosis negative, and two double negative controls), ZIEGLER et al. [54] have reported in humans that only $0.8 \%$ of type II alveolar epithelial cells expressed both ACE2 and TMPRSS2 genes. Further analysis of ciliated cells found that 5.3\% of these cells expressed both ACE2 and TMPRSS2 genes. In vitro models with SARS-CoV are consistent with this finding, as ciliated cells are preferentially targeted by this coronavirus [58]. Most intriguing is that ACE2 and TMPRSS2 gene-expressing cells were only identified in the HIV and tuberculosis double positive samples. These observations were replicated in the upper airways, with only a rare population of secretory epithelial cells ( $0.3 \%$ of this population) co-expressing ACE2 and TMPRSS2. The reported scRNAseq results are consistent with a focused analysis looking at only ACE2 gene expression in a variety of lung cell types [59]. Importantly, these elegant transcriptomic analyses confirm our observations in bulk tissue microarray datasets.

Consortium-based publicly available datasets represent another parallel approach to confirm our data. We have used the FANTOM5 dataset containing CAGE promoter activation data for 1866 primary cells, cell lines and tissue samples from humans [39] to examine the level of promoter activity for each candidate SARS-CoV-2 receptor gene. The FANTOM5 CAGE data provide an additional and complementary approach to quantifying gene expression, since a given gene's shared promoter can yield multiple transcripts at different expression levels, as well as being partially independent of any given transcript's half-life in the cell. In general, the promoter activity of ACE2 in airway-related tissues is low or absent; only a single sample originating from an adult lung yielded a normalised CAGE promoter expression level $>1$ TPM, while expression was observed in gut cells, consistent with known patterns of ACE2 expression [60]. Consistent with the microarray data, CD147 promoter activity is elevated relative to ACE2 across airway-related cells and tissues, although the relatively low CTSL promoter activity is incongruent with modest levels of gene expression.

The expression of genes does not always correlate with protein expression [49]. With this in mind, we performed combination proteomic analyses with immunoblot analyses. For our immunoblots, we used the human Calu-3 adenocarcinoma cell line, as this cell line is susceptible and permissive to SARS-CoV-2 infection and expresses ACE2, an observation we confirm [24, 29]. We also used primary human airway epithelial cells and the bronchial epithelial cell line (HBEC-6KT). We performed immunoblots for ACE2 and TMPRSS2 as these have been highlighted as interacting with SARS-CoV-2, while we probed CD147 as recent pre-clinical and clinical studies have provided proof of concept for this as a candidate SARS-CoV-2 receptor [26, 27]. Lastly, GRP78 was dominantly expressed throughout transcriptomic studies and was selected as a positive control, as previous expression has been confirmed in human airway epithelial cells [61]. Cathepsin L was excluded from the present analysis due to low promoter activity (figure 3), while ADAM17 was excluded as the proposed function in coronavirus infections is via ACE2 [5, 24], which was included in analysis. Immunoblot analysis with all antibodies revealed dominant bands of predicted molecular weight, with the anti-TMPRSS2 polyclonal antibody revealing additional minor bands in all cell samples examined. The identity of these other bands remains unclear and suggests downstream immunohistochemical analysis may be confounded by the specificity of this antibody. In contrast, antibodies for ACE2, CD147 and GRP78 were specific and could be used for immunohistochemistry without concerns of specificity. Interestingly, ACE2 protein could only be detected with a super-sensitive ECL solution and only in Calu-3 cells, suggesting absent protein in primary human airway epithelial cells and the HBEC-6KT cell line. Our data are consistent with previous immunoblots of primary human airway epithelial cells grown under submerged monolayer conditions using the same primary antibody, where ACE2 protein was absent, and only expressed under air-liquid interface culture conditions [62]. The 
observation that CD147 and GRP78 are also expressed in Calu-3 cells encourages further interrogation into these host proteins, as they may contribute to function of ACE2 and TMPRSS2 in SARS-CoV-2 binding and fusion in this cell type. Collectively, the profiling of antibodies by immunoblot of airway epithelial cells revealed distinct band patterns demonstrative of antibody specificity for ACE2, CD147 and GRP78, and to a lesser extent for TMPRSS2.

Immunohistochemical analysis has been performed for localisation of ACE2 and TMPRSS2 in human lung $[28,30]$. The observation of positive staining in human lung tissue for these proteins was not accompanied by companion immunoblot or complementary approaches to define the specificity of the antibody used [51]. In the absence of determination of antibody specificity, the historical data presented should be interpreted with caution. To address the issue of antibody specificity for immunohistochemical staining, we used the same antibodies we validated by immunoblot and confirmed findings using proteomics as an orthogonal, antibody independent, approach. We again focused on ACE2 and TMPRSS2 as these are candidate proteins important for SARS-CoV-2 infection of host cells. Our immunohistochemical staining patterns of ACE2 were consistent with transcriptional profiling and immunoblots with only one out of 98 human samples demonstrating rare staining in the airway and alveolar epithelium. Positive ACE2 staining in heart tissues and areas of lung microvasculature suggest our staining protocol was successful. These results directly contrast with those reported using antibodies that lacked validation for specificity [28, 30]. TMPRSS2 was expressed more frequently across all samples examined, with variability in the airway epithelium associated with history of smoking and/or COPD status. In contrast, CD147 expression was observed in airway epithelium of all samples. Similar to TMPRSS2, elevated CD147 expression was associated with history of smoking and/or COPD status, consistent with previous reports [50]. Our original GRP78 antibody selected for immunoblotting was not validated for immunohistochemistry. We therefore performed confirmatory GRP78 immunoblotting and immunohistochemistry with an additional antibody (HPA038845) and provided demonstration of expression in human airway epithelial cells in vitro and in situ. Importantly, it is well established that GRP78 resides in the endoplasmic reticulum (ER) under normal physiological conditions, where it acts as an ER-resident molecular chaperone to facilitate correct protein folding. However, under conditions of ER stress, including viral infection, a portion of ER-resident GRP78 relocates to the cell surface, where it may act as a viral co-receptor $[63,64]$. The presence of cell surface GRP78 has been reported in atherosclerotic plaques [65], prostate cancer [66] and kidney [67]. However, there are currently no commercially available anti-GRP78 antibodies that bind specifically to cell surface GRP78 and dual immunofluorescence is used to show co-localisation of cell surface GRP78 with an established surface receptor [65]. This drawback precludes our ability to perform accurate cell surface GRP78 immunohistochemistry on lung tissue to interrogate this concept further in the context of SARS-CoV-2 receptors. Utilisation of prostate cancer patient-derived GRP78 auto-antibodies that are specific for cell surface GRP78 [65] may be suitable on lung tissue for assessment of cell susceptibility to SARS-CoV-2 infection by GRP78.

Our study has several limitations that have not already been addressed. Our observation of differences in gene expression between upper and lower airways and along the airway tree were not corroborated at the protein level. It remains possible that entirely different protein expression profiles for the candidate molecules examined exist in the upper airway, presenting a different environment for SARS-CoV-2 interaction with the respiratory mucosa. Nasal pharyngeal swabs are capable of detecting SARS-CoV-2 virus [68] and this anatomical region is probably important for subsequent infection in the lower airways $[8,69]$. Related to this potential temporality of effect, it is possible that SARS-CoV-2 induces the expression of receptors on host cells following infection [54]. Our study is also limited by examining candidate molecules important in SARS-CoV-2 infection under basal conditions, in the absence of viral or environmental stimuli that may regulate gene transcription and protein translation.

SARS-CoV-2 infection and transmission has caused the global COVID-19 pandemic. An understanding of the receptors used by SARS-CoV-2 for host cell infection and the parallel characterisation in human samples is required to inform development of intervention strategies aimed at mitigating COVID-19. Our data demonstrate rare ACE2 protein expression in human airway epithelial cells in vitro and in situ, consistent with low ACE2 promoter activity and ACE2 gene expression in bronchial epithelial cells. We present confirmatory evidence for the presence of TMPRSS2, CD147 and GRP78 protein in vitro in airway epithelial cells and confirm broad in situ protein expression of CD147 and GRP78 in the respiratory mucosa. Due to the overwhelming evidence that the SARS virus interacts with ACE2, there are likely to be alternate mechanisms regulating ACE2 in the respiratory mucosa in the context of SARS-CoV-2 infection, and/or perhaps other co-receptors, beyond what is expressed under basal conditions at the protein level.

Acknowledgements: We would like to acknowledge Mary Jo Smith from the McMaster Immunology Research Centre Core Histology facility (McMaster University, Hamilton, ON, Canada) for her timely and professional expertise with antibody staining for immunohistochemistry. We would like to thank Sam Wadsworth (Aspect Biosystems, Vancouver, 
BC, Canada) and John McDonough (Yale University, New Haven, CT, USA) for their intellectual discussions around CD147 and airway epithelial cell biology. We would like to thank Charles Plessy (Okinawa Institute of Science and Technology, Onna, Japan) for his suggestion to analyse the FANTOM5 data. We would like to thank all of the personal and professional support from the authors' respective families (Candace Lee Bastedo, Julianne Meiko Hirota and Hana Lee Hirota) and research institutes and, most importantly, the frontline healthcare workers during the COVID-19 pandemic.

Conflict of interest: J.A. Aguiar has nothing to disclose. B.J-M. Tremblay has nothing to disclose. M.J. Mansfield has nothing to disclose. O. Woody has nothing to disclose. B. Lobb has nothing to disclose. A. Banerjee has nothing to disclose. A. Chandiramohan has nothing to disclose. N. Tiessen has nothing to disclose. Q. Cao has nothing to disclose. A. Dvorkin-Gheva has nothing to disclose. S. Revill has nothing to disclose. M.S. Miller has nothing to disclose C. Carlsten has nothing to disclose. L. Organ has nothing to disclose. C. Joseph has nothing to disclose. A. John has nothing to disclose. P. Hanson has nothing to disclose. R. Austin has a patent US7524826B2 issued to McMaster University and Hamilton Health Sciences Corp., McMaster University. B.M. McManus has nothing to disclose. G. Jenkins reports grants from AstraZeneca, Biogen, Galecto and GlaxoSmithKline; personal fees from Boehringer Ingelheim, Daewoong, Galapagos, Heptares, Promedior and Roche; grants and personal fees from Pliant; non-financial support from Redx and NuMedii; and is trustee for Action for Pulmonary Fibrosis, outside the submitted work. K. Mossman has nothing to disclose. K. Ask has nothing to disclose. A.C. Doxey has nothing to disclose. J.A. Hirota has nothing to disclose.

Support statement: This work was supported by start-up funds from J.A. Hirota and a CIHR grant from K. Mossman. J.A. Hirota is supported by the Canada Research Chairs program and an Ontario Early Researcher Award. A.C. Doxey is supported by NSERC and an Ontario Early Researcher Award. Funding information for this article has been deposited with the Crossref Funder Registry.

\section{References}

1 Bauch CT, Lloyd-Smith JO, Coffee MP, et al. Dynamically modeling SARS and other newly emerging respiratory illnesses: past, present, and future. Epidemiology 2005; 16: 791-801.

2 Centers for Disease Control and Prevention, National Center for Immunization and Respiratory Diseases (NCIRD). SARS Basics Fact Sheet. www.cdc.gov/sars/about/fs-sars.html Date last updated: 6 December 2017.

3 Peiris JSM, Yuen KY, Osterhaus ADME, et al. The severe acute respiratory syndrome. N Engl J Med 2003; 349: 2431-2441.

4 Munster VJ, Koopmans M, van Doremalen N, et al. A novel coronavirus emerging in China - key questions for impact assessment. N Engl J Med 2020; 382: 692-694.

5 Zhou P, Lou YX, Wang XG, et al. A pneumonia outbreak associated with a new coronavirus of probable bat origin. Nature 2020; 579: 270-273.

6 Cascella M, Rajnik M, Cuomo A, et al. Features, Evaluation and Treatment Coronavirus (COVID-19). Treasure Island, StatPearls Publishing, 2020.

$7 \mathrm{Gu} \mathrm{J}$, Korteweg C. Pathology and pathogenesis of severe acute respiratory syndrome. Am J Pathol 2007; 170: $1136-1147$

8 Zou L, Ruan F, Huang M, et al. SARS-CoV-2 viral load in upper respiratory specimens of infected patients. N Engl J Med 2020; 382: 1177-1179.

9 Wölfel R, Corman VM, Guggemos W, et al. Virological assessment of hospitalized patients with COVID-2019. Nature 2020; 581: 465-469.

$10 \mathrm{Lu}$ R, Zhao X, Li J, et al. Genomic characterisation and epidemiology of 2019 novel coronavirus: implications for virus origins and receptor binding. Lancet 2020; 395: 565-574.

11 Letko M, Marzi A, Munster V. Functional assessment of cell entry and receptor usage for SARS-CoV-2 and other lineage B betacoronaviruses. Nat Microbiol 2020; 5: 562-569.

12 Yan R, Zhang Y, Li Y, et al. Structural basis for the recognition of the SARS-CoV-2 by full-length human ACE2. Science 2020; 367: 1444-1448.

13 Wrapp D, Wang N, Corbett KS, et al. Cryo-EM structure of the 2019-nCoV spike in the prefusion conformation. Science 2020; 367: 1260-1263.

$14 \mathrm{Li}$ W, Moore MJ, Vasllieva N, et al. Angiotensin-converting enzyme 2 is a functional receptor for the SARS coronavirus. Nature 2003; 426: 450-454

15 Glowacka I, Bertram S, Muller MA, et al. Evidence that TMPRSS2 activates the severe acute respiratory syndrome coronavirus spike protein for membrane fusion and reduces viral control by the humoral immune response. J Virol 2011; 85: 4122-4134.

16 Shulla A, Heald-Sargent T, Subramanya G, et al. A transmembrane serine protease is linked to the severe acute respiratory syndrome coronavirus receptor and activates virus entry. J Virol 2011; 85: 873-882.

17 Chen YW, Lee MS, Lucht A, et al. TMPRSS2, a serine protease expressed in the prostate on the apical surface of luminal epithelial cells and released into semen in prostasomes, is misregulated in prostate cancer cells. Am J Pathol 2010; 176: 2986-2996.

18 Haga S, Nagata N, Okamura T, et al. TACE antagonists blocking ACE2 shedding caused by the spike protein of SARS-CoV are candidate antiviral compounds. Antiviral Res 2010; 85: 551-555.

19 Iwata M, Silva Enciso JE, Greenberg BH. Selective and specific regulation of ectodomain shedding of angiotensin-converting enzyme 2 by tumor necrosis factor alpha-converting enzyme. Am J Physiol Cell Physiol 2009; 297: C1318-C1329.

20 Heurich A, Hofmann-Winkler H, Gierer S, et al. TMPRSS2 and ADAM17 cleave ACE2 differentially and only proteolysis by TMPRSS2 augments entry driven by the severe acute respiratory syndrome coronavirus spike protein. J Virol 2014; 88: 1293-1307.

21 Simmons G, Gosalia DN, Rennekamp AJ, et al. Inhibitors of cathepsin L prevent severe acute respiratory syndrome coronavirus entry. Proc Natl Acad Sci USA 2005; 102: 11876-11881. 
22 Chen Z, Mi L, Xu J, et al. Function of HAb18G/CD147 in invasion of host cells by severe acute respiratory syndrome coronavirus. J Infect Dis 2005; 191: 755-760.

23 Chu $\mathrm{H}$, Chan CM, Zhang X, et al. Middle East respiratory syndrome coronavirus and bat coronavirus HKU9 both can utilize GRP78 for attachment onto host cells. J Biol Chem 2018; 293: 11709-11726.

24 Hoffmann M, Kleine-Weber H, Schroeder S, et al. SARS-CoV-2 cell entry depends on ACE2 and TMPRSS2 and is blocked by a clinically proven protease inhibitor. Cell 2020; 181: 271-280.

25 Ibrahim IM, Abdelmalek DH, Elshahat ME, et al. COVID-19 spike-host cell receptor GRP78 binding site prediction. J Infect 2020; 80: 554-562.

26 Wang K, Chen W, Zhou YS, et al. SARS-CoV-2 invades host cells via a novel route: CD147-spike protein. bioRxiv 2020; preprint [https://doi.org/10.1101/2020.03.14.988345].

27 Bian H, Zheng ZH, Wei D, et al. Meplazumab treats COVID-19 pneumonia: an open-labelled, concurrent controlled add-on clinical trial. medRxiv 2020; preprint [https://doi.org/10.1101/2020.03.21.20040691].

28 Hamming I, Timens W, Bulthuis MLC, et al. Tissue distribution of ACE2 protein, the functional receptor for SARS coronavirus. A first step in understanding SARS pathogenesis. J Pathol 2004; 203: 631-637.

29 Tseng CTK, Tseng J, Perrone L, et al. Apical entry and release of severe acute respiratory syndrome-associated coronavirus in polarized Calu-3 lung epithelial cells. J Virol 2005; 79: 9470-9479.

30 Bertram S, Heurich A, Lavender $\mathrm{H}$, et al. Influenza and SARS-coronavirus activating proteases TMPRSS2 and HAT are expressed at multiple sites in human respiratory and gastrointestinal tracts. PLoS One 2012; 7: e35876.

31 Kim MS, Pinto SM, Getnet D, et al. A draft map of the human proteome. Nature 2014; 509: 575-581.

32 Foster MW, Gwinn WM, Kelly FL, et al. Proteomic analysis of primary human airway epithelial cells exposed to the respiratory toxicant diacetyl. J Proteome Res 2017; 16: 538-549.

33 Giovannini-Chami L, Marcet B, Moreilhon C, et al. Distinct epithelial gene expression phenotypes in childhood respiratory allergy. Eur Respir J 2012; 39: 1197-1205.

34 Raman T, O'Connor TP, Hackett NR, et al. Quality control in microarray assessment of gene expression in human airway epithelium. BMC Genomics 2009; 10: 493.

35 Davis S, Meltzer PS. GEOquery: a bridge between the Gene Expression Omnibus (GEO) and BioConductor. Bioinformatics 2007; 23: 1846-1847.

36 Huber W, Carey VJ, Gentleman R, et al. Orchestrating high-throughput genomic analysis with Bioconductor. Nat Methods 2015; 12: 115-121.

37 Johnson WE, Li C, Rabinovic A. Adjusting batch effects in microarray expression data using empirical Bayes methods. Biostatistics 2007; 8: 118-127.

38 Leek JT, Johnson WE, Parker HS, et al. The sva package for removing batch effects and other unwanted variation in high-throughput experiments. Bioinformatics 2012; 28: 882-883.

39 Forrest ARR, Kawaji H, Rehli M, et al. A promoter-level mammalian expression atlas. Nature 2014; 507: 462-470.

40 Abugessaisa I, Noguchi S, Hasegawa A, et al. FANTOM5 CAGE profiles of human and mouse reprocessed for GRCh38 and GRCm38 genome assemblies. Sci Data 2017; 4: 170107.

41 Severin J, Lizio M, Harshbarger J, et al. Interactive visualization and analysis of large-scale sequencing datasets using ZENBU. Nat Biotechnol 2014; 32: 217-219.

42 Butler A, Hoffman P, Smibert P, et al. Integrating single-cell transcriptomic data across different conditions, technologies, and species. Nat Biotechnol 2018; 36: 411-420.

43 Habermann AC, Gutierrez AJ, Bui LT, et al. Single-cell RNA sequencing reveals profibrotic roles of distinct epithelial and mesenchymal lineages in pulmonary fibrosis. Sci Adv 2020; 6: eaba1972.

44 Huff RD, Rider CF, Yan D, et al. Inhibition of ABCC4 potentiates combination beta agonist and glucocorticoid responses in human airway epithelial cells. J Allergy Clin Immunol 2018; 141: 1127-1130.e5.

45 Hirota JA, Gold MJ, Hiebert PR, et al. The nucleotide-binding domain, leucine-rich repeat protein 3 inflammasome/IL-1 receptor I axis mediates innate, but not adaptive, immune responses after exposure to particulate matter under $10 \mu \mathrm{m}$. Am J Respir Cell Mol Biol 2015; 52: 96-105.

46 Hirota JA, Marchant DJ, Singhera GK, et al. Urban particulate matter increases human airway epithelial cell IL-1 $\beta$ secretion following scratch wounding and H1N1 influenza A exposure in vitro. Exp Lung Res 2015; 41: 353-362.

47 Ramirez RD, Sheridan S, Girard L, et al. Immortalization of human bronchial epithelial cells in the absence of viral oncoproteins. Cancer Res 2004; 64: 9027-9034.

48 Gürtler A, Kunz N, Gomolka M, et al. Stain-Free technology as a normalization tool in Western blot analysis. Anal Biochem 2013; 433: 105-111.

49 Vogel C, Marcotte EM. Insights into the regulation of protein abundance from proteomic and transcriptomic analyses. Nat Rev Genet 2012; 13: 227-232.

50 Jouneau S, Khorasani N, De Souza P, et al. EMMPRIN (CD147) regulation of MMP-9 in bronchial epithelial cells in COPD. Respirology 2011; 16: 705-712.

51 Uhlen M, Bandrowski A, Carr S, et al. A proposal for validation of antibodies. Nat Methods 2016; 13: 823-827.

52 Donoghue $\mathrm{M}$, Hsieh F, Baronas E, et al. A novel angiotensin-converting enzyme-related carboxypeptidase (ACE2) converts angiotensin I to angiotensin 1-9. Circ Res 2000; 87: E1-E9.

53 Burrell LM, Risvanis J, Kubota E, et al. Myocardial infarction increases ACE2 expression in rat and humans. Eur Heart J 2005; 26: 1142-1143.

54 Ziegler CGK, Allon SJ, Nyquist SK, et al. SARS-CoV-2 receptor ACE2 is an interferon-stimulated gene in human airway epithelial cells and is detected in specific cell subsets across tissues. Cell 2020; 181: 1016-1035.

55 Leung JM, Yang CX, Tam A, et al. ACE-2 expression in the small airway epithelia of smokers and COPD patients: implications for COVID-19. Eur Respir J 2020; 55: 2000688.

56 Schiller HB, Montoro DT, Simon LM, et al. The human lung cell atlas: a high-resolution reference map of the human lung in health and disease. Am J Respir Cell Mol Biol 2019; 61: 31-41.

57 Plasschaert LW, Žilionis R, Choo-Wing R, et al. A single-cell atlas of the airway epithelium reveals the CFTR-rich pulmonary ionocyte. Nature 2018; 560: 377-381.

58 Sims AC, Baric RS, Yount B, et al. Severe acute respiratory syndrome coronavirus infection of human ciliated airway epithelia: role of ciliated cells in viral spread in the conducting airways of the lungs. J Virol 2005; 79: 15511-15524. 
Qi F, Qian S, Zhang S, et al. Single cell RNA sequencing of 13 human tissues identify cell types and receptors of human coronaviruses. Biochem Biophys Res Commun 2020; 526: 135-140.

60 Hikmet F, Méar L, Edvinsson A, et al. The protein expression profile of ACE2 in human tissues. Mol Syst Biol 2020; 16: e9610.

61 Aksoy MO, Kim V, Cornwell WD, et al. Secretion of the endoplasmic reticulum stress protein, GRP78, into the BALF is increased in cigarette smokers. Respir Res 2017; 18: 78.

62 Jia HP, Look DC, Shi L, et al. ACE2 receptor expression and severe acute respiratory syndrome coronavirus infection depend on differentiation of human airway epithelia. J Virol 2005; 79: 14614-14621.

63 Gonzalez-Gronow M, Selim MA, Papalas J, et al. GRP78: a multifunctional receptor on the cell surface. Antioxid Redox Signal 2009; 11: 2299-2306.

64 Zhang Y, Liu R, Ni M, et al. Cell surface relocalization of the endoplasmic reticulum chaperone and unfolded protein response regulator GRP78/BiP. J Biol Chem 2010; 285: 15065-15075.

65 Crane ED, Al-Hashimi AA, Chen J, et al. Anti-GRP78 autoantibodies induce endothelial cell activation and accelerate the development of atherosclerotic lesions. JCI Insight 2018; 3: e99363.

66 Al-Hashimi AA, Lebeau P, Majeed F, et al. Autoantibodies against the cell surface-associated chaperone GRP78 stimulate tumor growth via tissue factor. J Biol Chem 2017; 292: 21180-21192.

67 Van Krieken R, Mehta N, Wang T, et al. Cell surface expression of 78-kDa glucose-regulated protein (GRP78) mediates diabetic nephropathy. J Biol Chem 2019; 294: 7755-7768.

68 Wang W, Xu Y, Gao R, et al. Detection of SARS-CoV-2 in different types of clinical specimens. JAMA 2020; 323: $1843-1844$.

69 Hou YJ, Okuda K, Edwards CE, et al. SARS-CoV-2 reverse genetics reveals a variable infection gradient in the respiratory tract. Cell 2020; 182: 429-446. 